\title{
EL MANEJO DE LOS ANIMALES EN INSTALACIONES DE REMATES FERIAS Y SU IMPACTO SOBRE EL BIENESTAR ANIMAL Y LA CALIDAD DE LA CARNE
}

\section{EVALUACIÓN DEL TRATO A LOS ANIMALES DURANTE EL MANEJO PREVIO AL REMATE EN INSTALACIONES DE REMATES FERIAS}

\author{
THOMAS, J. ${ }^{1} ;$ ASTESANA, E. ${ }^{2} \&$ URSO, R. ${ }^{2}$
}

\begin{abstract}
RESUMEN
En Argentina no hay datos publicados que permitan reconocer los efectos del proceso de comercialización en Ferias, sobre el bienestar de los animales y calidad de la carne. El propósito del trabajo es evaluar el trato de los animales durante las operaciones previas al remate (descarga, arreo, separación y encierro en corrales), comparando ferias con programa de aseguramiento de la calidad orientado al bienestar animal, con aquellos en donde se realiza el manejo tradicional, y evaluar su impacto sobre el bienestar de los animales y posibles efectos sobre la calidad de la carne. Se realizaron controles en 6 instalaciones de remates ferias de la Provincia de Santa Fe durante 6 meses, registrando 3823 animales. Se demuestra la importancia de implementar un Sistema de aseguramiento de la calidad para demostrar el cumplimiento de los requisitos del bienestar animal y reducir el impacto sobre la calidad de la carne obtenida.

Palabras claves: bienestar animal, comercialización, remates ferias.
\end{abstract}

\section{SUMMARY}

Animal management in saleyards and its impact on animal welfare and meat quality. Evaluation of animals handling prior to the auction on saleyard facilities.

In Argentina there are no published data to recognize the effects of the commercialization process, on animal welfare and meat quality. The purpose of this study was to evaluate animals handling during the pre-auction (download, herding, separation and confinement in pens), comparing saleyards

1.- Departamento Producción Animal, Facultad de Ciencias Agrarias (UNL). Kreder 2805. Esperanza, provincia de Santa Fe. Email: jthomas@fca.unl.edu.ar

2.- Estudiantes de la FCA (UNL).

Manuscrito recibido el 25 de febrero de 2011 y aceptado para su publicación el 27 de abril de 2011 . 